\title{
Physical Exercise with Multicomponent Cognitive Intervention for Older Adults with Alzheimer's Disease: A 6-Month Randomized Controlled Trial
}

\author{
Min-Ji Kima Chang-Wan Han ${ }^{a}$ c Kyoung-Youn Min ${ }^{d}$ Chae-Yoon Cho ${ }^{a}$ \\ Chae-Won Lee $^{a}$ Yoshiko Ogawa ${ }^{a}$ Etsuro Mori ${ }^{b}$ Masahiro Kohzukia \\ ${ }^{a}$ Department of Internal Medicine and Rehabilitation Science, and ${ }^{b}$ Division of Behavioral \\ Neurology and Cognitive Neuroscience, Tohoku University Graduate School of Medicine, \\ Sendai, and 'Faculty of Education, University of the RyuKyus, Okinawa, Japan; \\ dDobong Silver Center, Miral Welfare Foundation, Seoul, Republic of Korea
}

Key Words

Alzheimer's disease $\cdot$ Cognition $\cdot$ Nonpharmacologic treatment $\cdot$ Physical activity

\section{Abstract}

Aims: This study aimed to investigate the effect of 6 -month physical exercise with a multicomponent cognitive program (MCP) on the cognitive function of older adults with moderate to severe Alzheimer's disease (AD). Methods: We included 33 participants with $A D$ in a 6 -month randomized controlled trial. The intervention group participated in physical exercise and received a MCP. The control group received only the MCP. Before and after the intervention, cognitive outcomes were assessed using the Alzheimer's Disease Assessment ScaleCognitive Subscale (ADAS-cog), Mini-Mental State Examination, and the Clock Drawing Test. Physical performance was evaluated by exercise time, the number of pedal rotation, total load, grip strength, and the Berg Balance Scale (BBS). Results: In all cognitive measures, there were no significant improvements between the two groups after 6 months in the baseline value-adjusted primary analysis. However, the ADAS-cog score was significantly lower between the two groups in secondary analysis adjusted for baseline value, age, sex, and education years. All physical outcomes were significantly higher in the intervention group except for total load compared with baseline measurements. Conclusion: This study indicates that it is possible to improve cognitive function in older adults with moderate to severe AD through 6 -month physical exercise with a multicomponent cognitive intervention. 
Kim et al.: Physical Exercise with Multicomponent Cognitive Intervention for Older Adults with Alzheimer's Disease: A 6-Month Randomized Controlled Trial

\section{Introduction}

Alzheimer's disease (AD) is a severe chronic neurodegenerative disease characterized by progressive cognitive impairment, functional decline, and neuropsychiatric symptoms [1]. Many clinical trials of AD drug treatment have failed to show a drug or placebo difference or have had unacceptable toxicity levels [2]. Even AD drug treatments initiated earlier, at around 6 months, have not shown significant differences in cognitive function, physical function, behavioral problems, and clinical status [3].

Some studies indicate that nonpharmacological interventions using a wide range of approaches have demonstrated efficacy in treating cognitive symptoms with no adverse effects $[4,5]$. Of these interventions, physical activity constitutes an effective intervention in neurodegenerative diseases, attenuating or limiting their progression [6, 7]. However, recent studies have suggested that there is no benefit from physical activity on cognitive function [8] or on neuropsychiatric symptoms or depression [9]. Furthermore, there are many randomized controlled trial (RCT) reports of the effect of physical activity on cognitive function in individuals with mild to moderate AD [10-14], but few RCTs have assessed the effect of physical activity in patients with moderate to severe AD [15-17]. None of these studies has found significant differences in any of the cognitive outcome measures compared with a control group. Therefore, the effect of physical activity on cognitive functioning in individuals with moderate to severe AD has not been explored.

From another perspective, it has been proposed that a combination of nonpharmacological interventions is more effective for inducing neural and cognitive benefits rather than a single intervention [18]. Only a few RCTs have investigated the effect of a combination of cognitive interventions with no physical exercise on individuals with mild to moderate AD; those studies showed no evidence of effectiveness on cognitive function $[19,20]$. Furthermore, there has not been an investigation of the effects of a combination of physical exercise with cognitive interventions on individuals with moderate to severe AD.

The aims of this study were to examine the effect of 6-month physical exercise with a multicomponent cognitive program (MCP) on the cognitive function of older adults with moderate to severe AD. It was hypothesized that physical exercise with a MCP would result in better cognitive function than MCP alone.

\section{Materials and Methods}

\section{Participants}

This study was a single-blind 6-month RCT conducted from December 2014 to June 2015. Participants included older adults with moderate to severe AD living in a nursing home in Seoul, Republic of Korea. A total of 119 nursing home residents were contacted. Of these, 50 participants with AD met the inclusion criteria stipulated below. Written consent was obtained from all participants and their caregivers or their legal guardians. This study was conducted according to the declaration of Helsinki, and the protocol was approved by the Ethics Committee of the Tohoku University Graduate School of Medicine. The trial registration number is 2014-1-429.

\section{Inclusion Criteria}

Inclusion in the study was dependent on (i) a diagnosis of AD by a neurologist, (ii) living in a nursing home for at least 3 months, (iii) moderate to severe $\mathrm{AD}$ as determined by a baseline Mini-Mental State Examination (MMSE) score of $\leq 20$ [21], and (iv) informed consent. Fifty participants were screened for study entry. Of these, 12 participants (24\%) 
Kim et al.: Physical Exercise with Multicomponent Cognitive Intervention for Older Adults with Alzheimer's Disease: A 6-Month Randomized Controlled Trial

did not meet the inclusion criteria. Six had severe cognitive impairment with loss of communication skills, 2 had vascular dementia, and 4 refused to participate in this study (fig. 1).

\section{Randomization}

Thirty-eight participants were eligible and were randomized to the study intervention using an opaque sealed envelope [22]. The Kohzuki Exercise Program with MCP (KEP+MCP) or a single MCP was written down on paper by the researcher and put into each opaque envelope. A total of 38 envelopes were then shuffled by staff who were not involved with this study. The participant then chose an envelope and opened it. Each of the two groups consisted of 19 participants, who were randomly assigned to KEP+MCP or a single MCP.

\section{Intervention}

The KEP consisted of 60 min of supervised exercise sessions 5 times a week for 6 months: 15 min of warm-up and stretching, 30 min of lower-limb aerobic exercise using a TERASUERUGO $^{\circledR}$ (Showa Denki Co., Ltd., Osaka, Japan), and 15 min of cool-down and relaxation. Intensity of exercise was a heart rate of $40-60 \%$ of the maximum (Borg scale scores of 1113). The KEP was supervised by a physical therapist.

All participants in the KEP+MCP and MCP groups received the multicomponent intervention that consisted of music therapy [23], art therapy [24], horticulture therapy [25], handicraft [26], recreational therapy [27], stretching [28], laughing therapy [29], and activity therapy [30]. Each MCP intervention was conducted by professional therapists for 60 min per session twice a day, 5 days per week for 6 months. All MCP interventions were supervised by two caregivers per each group. Since the participants were residing in a facility for long-term care, their participation rate was $100 \%$. Each therapy was given equally to the KEP+MCP group and the MCP group quantitatively and qualitatively. In this study, a placebo group could not be included because it was not permitted within the facility.

\section{Outcome Measures}

Cognitive function was the primary outcome measure. General cognitive function was evaluated using the Korean version of the Alzheimer's Disease Assessment Scale-Cognitive Subscale (ADAS-cog) [31] and the MMSE [32]. The scoring range for the ADAS-cog is from 0 to 70, higher scores indicating greater cognitive impairment. MMSE scores range from 0 to 30, higher scores indicating greater cognitive function. The Clock Drawing Test (CDT) was performed to evaluate executive function [33]. Scores range from 0 to 10, higher scores indicating intact executive function. All cognitive measures were administered at baseline and after 6 months $(6 \mathrm{M})$.

Physical function was the secondary outcome measure. The pedal power consisted of 7 steps from 10 to $70 \mathrm{~W}$, the number of pedal rotations and exercise time was recorded to measure the total load. The pedal power was based on the Borg scale scores of 11-13. The total load was calculated as follows: (the pedal power $(\mathrm{W}) \times$ the number of pedal rotation)/ exercise time (s). Grip strength was measured in the dominant hand with a standard handgrip dynamometer (KS-301; LAVISEN Co., Ltd., Gyeonggi-do, Republic of Korea). Each participant stood upright with their arm vertical and the dynamometer close to their body. They were asked to put maximum force on the dynamometer twice using their dominant hand. The maximum value was recorded in kilograms. Finally, balance was assessed using the Berg Balance Scale (BBS) [34]. All physical measures were administered at baseline, after 1 month $(1 \mathrm{M})$, after 3 months $(3 \mathrm{M})$, and after 6 months $(6 \mathrm{M})$. 
Kim et al.: Physical Exercise with Multicomponent Cognitive Intervention for Older Adults with Alzheimer's Disease: A 6-Month Randomized Controlled Trial

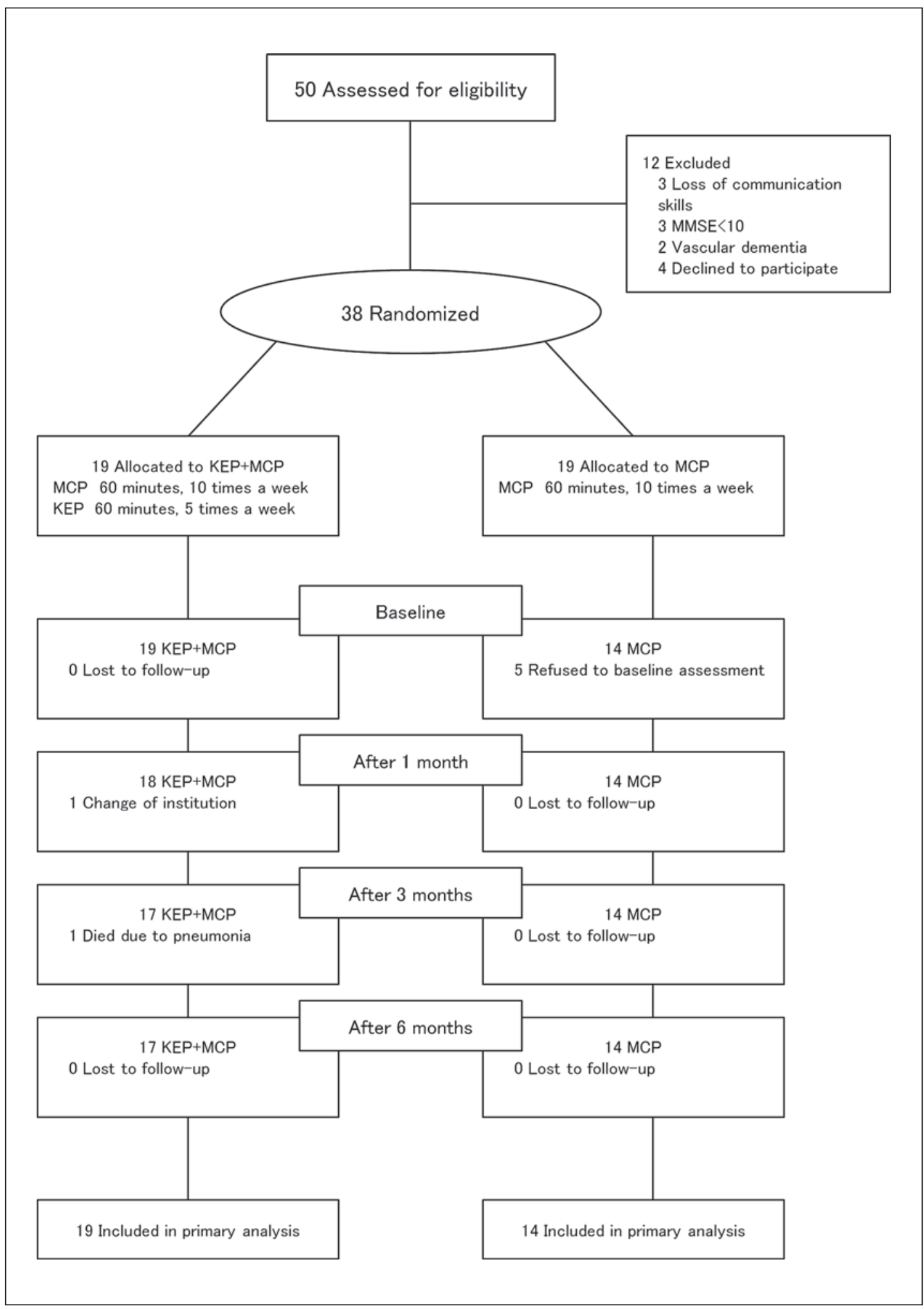

Fig. 1. Study flow diagram. Thirty-eight participants were randomized to participate in the KEP+MCP and MCP only. Of the 19 participants in the KEP+MCP group, 1 participant died of pneumonia and 1 participant dropped out due to change of institution. Of the 19 participants in the MCP group, 5 participants refused to undergo baseline assessment. Thirty-three participants were assessed at 6 months after the intervention: 19 in the KEP+MCP group and 14 in the MCP group. 
Table 1. Baseline demographic characteristics

\begin{tabular}{|c|c|c|c|c|}
\hline & $\begin{array}{l}\text { Total } \\
(\mathrm{n}=33)\end{array}$ & $\begin{array}{l}\text { KEP+MCP } \\
(n=19)\end{array}$ & $\begin{array}{l}\text { MCP } \\
(n=14)\end{array}$ & $\mathrm{p}$ value \\
\hline Mean age $\pm S D$, years & $81.5 \pm 6.6$ & $81.9 \pm 7.0$ & $80.9 \pm 6.1$ & 0.53 \\
\hline Females, $\mathrm{n}$ & $25(75.8)$ & $13(68.4)$ & 12 (85.7) & 0.49 \\
\hline Mean height $\pm \mathrm{SD}, \mathrm{cm}$ & $155.6 \pm 7.2$ & $157.9 \pm 7.4$ & $152.5 \pm 5.7$ & 0.06 \\
\hline Mean weight \pm SD, $\mathrm{kg}$ & $55.9 \pm 8.9$ & $55.1 \pm 9.9$ & $56.9 \pm 7.7$ & 0.23 \\
\hline Mean educational level $\pm \mathrm{SD}$, years & $7.7 \pm 5.1$ & $7.4 \pm 5.8$ & $8.0 \pm 4.2$ & 0.43 \\
\hline \multicolumn{5}{|l|}{ Walking state, n } \\
\hline Using a wheelchair & $21(63.6)$ & $10(52.6)$ & $11(78.6)$ & 0.16 \\
\hline Using a walker & $7(21.2)$ & $5(26.3)$ & $2(14.3)$ & 0.96 \\
\hline Walking independently & $5(15.2)$ & $4(21.1)$ & $1(7.1)$ & 0.96 \\
\hline \multicolumn{5}{|l|}{ Complications, $\mathrm{n}$} \\
\hline Hypertension & $29(87.9)$ & $17(89.5)$ & $12(85.7)$ & 0.57 \\
\hline Diabetes & $16(48.5)$ & $11(57.9)$ & $5(28.6)$ & 0.33 \\
\hline Stroke & $15(45.5)$ & $10(52.6)$ & $5(35.7)$ & 0.33 \\
\hline Depression & $4(12.1)$ & $3(15.8)$ & $1(7.1)$ & 0.57 \\
\hline Cancer & $2(6.1)$ & $1(5.3)$ & $1(7.1)$ & 0.70 \\
\hline Eye diseases & $10(30.3)$ & $7(36.8)$ & $3(21.4)$ & 0.08 \\
\hline Fracture & $9(27.3)$ & $6(31.6)$ & $3(21.4)$ & 0.22 \\
\hline Arthritis & $8(24.2)$ & $3(15.8)$ & $5(35.7)$ & 0.66 \\
\hline Cardiac disease & $4(12.1)$ & $3(15.8)$ & $1(7.1)$ & 0.57 \\
\hline History of head trauma, $n$ & $1(3.0)$ & $1(5.3)$ & $0(0.0)$ & 0.34 \\
\hline Mean MMSE \pm SD & $14.8(4.4)$ & $13.4(4.2)$ & $16.6(4.0)$ & 0.03 \\
\hline \multicolumn{5}{|l|}{ AD drugs, $n$} \\
\hline Donepezil & $5(15.2)$ & $2(10.5)$ & $3(21.4)$ & 0.39 \\
\hline Memantine & $3(9.1)$ & $2(10.5)$ & $1(7.1)$ & 0.74 \\
\hline Galantamine & $1(3.0)$ & $1(5.3)$ & $0(0.0)$ & 0.38 \\
\hline
\end{tabular}

Values in parentheses are percentages. The $\mathrm{p}$ value indicates the baseline difference between the groups. $\mathrm{SD}=$ Standard deviation .

\section{Statistical Analyses}

All analyses of outcomes covered the full analysis dataset, because the intention-to-treat set considered all dropout cases as untreated cases [35]. The full analysis dataset was defined as the set of participants who were randomly assigned to either of the two groups and who had at least one valid cognitive assessment after the KEP+MCP or MCP in this study.

We compared the changes from baseline using the Wilcoxon signed rank test, and the differences of cognitive change between the groups using analysis of covariance (ANCOVA). The outcome variable 6 months after the randomization was regarded in the model as a dependent variable. The baseline value of the outcome variable was included as a covariate in the primary analysis. The baseline value, age, sex, and education years were included as covariates in the secondary analysis. Finally, the study group (either the KEP+MCP or MCP group) was included as a categorical variable. The $\mathrm{p}$ values in all of the tests were two-sided, and $p$ values $<0.05$ were considered significant. All of the statistical analyses were performed with SPSS 17.0.0 for Windows.

\section{Results}

Demographic and Baseline Characteristics

Five participants withdrew from the MCP group after a few weeks. Their withdrawal was due to loss of motivation. Among the 38 participants initially present, only 33 completed the 
Table 2. Baseline scores and changes in physical functions

\begin{tabular}{lccccc}
\hline \multicolumn{7}{c}{ Mean change in score from baseline \pm SD } & \\
\cline { 2 - 6 } & baseline & $1 \mathrm{M}$ & $3 \mathrm{M}$ & $6 \mathrm{M}$ & $\mathrm{p}$ value \\
\hline Exercise time & $207.7 \pm 183.3$ & $351.3 \pm 277.2$ & $491.3 \pm 289.8$ & $656.8 \pm 315.5$ & $<0.0001$ \\
Pedal rotation & $97.7 \pm 89.9$ & $190.1 \pm 186.6$ & $258.3 \pm 253.7$ & $285.8 \pm 197.5$ & 0.004 \\
Total load & $6.3 \pm 7.5$ & $10.2 \pm 13.7$ & $10.8 \pm 12.7$ & $10.0 \pm 6.8$ & 0.06 \\
Grip strength & $7.9 \pm 5.9$ & $8.2 \pm 5.1$ & $11.4 \pm 6.3$ & $11.8 \pm 7.7$ & 0.02 \\
BBS & $28.2 \pm 17.6$ & $28.2 \pm 17.6$ & $25.7 \pm 18.8$ & $21.5 \pm 17.3$ & 0.04 \\
\hline
\end{tabular}

The $\mathrm{p}$ value was determined by one-way analysis of variance. SD = Standard deviation.

whole series of initial and final assessments (fig. 1). Table 1 shows the demographic data and baseline variables based on analysis of the full dataset. No significant differences were shown between the two groups at baseline except for the MMSE scores, which were significantly different between the groups at baseline. The mean age \pm standard deviation of all participants was $81.5 \pm 6.6$ years. Sixty-four percent of all participants used a wheelchair, $21 \%$ used a walker, and $15 \%$ walked independently without any assistive devices.

\section{Assessment of Cognitive Function}

There were significant within-group differences for the ADAS-cog score but not for the MMSE and CDT scores in the KEP+MCP group over the 6 months of the intervention. In contrast, there were no significant differences in the MCP group for all cognitive measures over the 6 months of the intervention.

None of the cognitive measures improved significantly after 6 months in the KEP+MCP group compared with the MCP group in the ANCOVA analysis adjusted for only the baseline value (ADAS-cog: $F=3.24, p=0.08$, MMSE: $F=0.66, p=0.80, C D T: F=3.01, p=0.09$ ). However, the ADAS-cog score was significantly lower after 6 months in the KEP+MCP group than in the MCP group in the ANCOVA analysis adjusted for baseline value, age, sex, and educational years $(F=5.20, p=0.03)$, whereas MMSE and CDT scores were not significantly improved between the groups (MMSE: $F=0.00, p=0.98, C D T: F=1.95, p=0.17$ ) (fig. 2 ).

\section{Assessment of Physical Function}

Table 2 shows changes in physical functions over the 6 months in the KEP+MCP group. Exercise time was significantly increased at 6 months $(p<0.0001)$. The number of pedal rotation was significantly increased at 6 months $(\mathrm{p}<0.004)$. Total load tended towards an increase at 6 months $(\mathrm{p}<0.06)$. Grip strength was significantly increased at 6 months $(\mathrm{p}<$ $0.02)$. Finally, BBS was significantly increased at 6 months $(\mathrm{p}<0.04)$.

\section{Discussion}

This study showed that a 6-month physical exercise with a MCP was associated with better cognitive function compared with only a MCP. Furthermore, the KEP+MCP group experienced significant differences in ADAS-cog and MMSE score compared with baseline scores, whereas the MCP group showed no significant differences in any cognitive outcome.

This study is the first RCT to evaluate the effect of a physical exercise with multicomponent cognitive intervention on cognitive function. We demonstrated that a combination of 
Kim et al.: Physical Exercise with Multicomponent Cognitive Intervention for Older Adults with Alzheimer's Disease: A 6-Month Randomized Controlled Trial

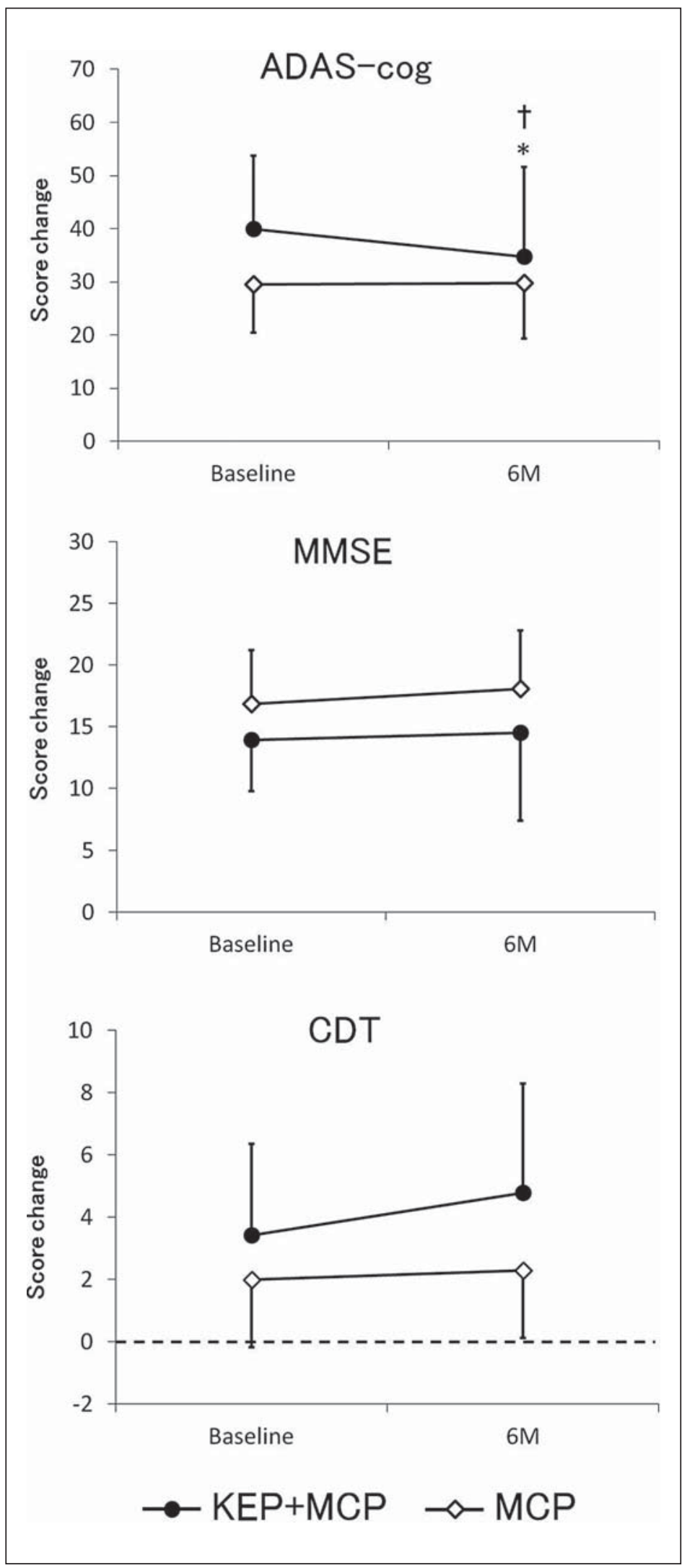

Fig. 2. Changes in cognitive functions from baseline. Mean score change from baseline for ADAScog, MMSE, and CDT to 6 months (6M). A different symbol is used for each group: the black dot indicates the KEP+MCP group and the white dot indicates the MCP group. Each point represents a mean value, and each error bar represents a standard deviation. * $\mathrm{p}<0.05$ compared with baseline; ${ }^{\dagger} \mathrm{p}<0.05$ compared with the MCP group adjusted for baseline value, age, sex, and education years. 
KEP and MCP may have cognitive benefits in people with moderate to severe AD. Fifty-three percent of the participants reported spending time sitting in a wheelchair every day, and $26 \%$ were walking with a walker because of a complicated fracture or hemiplegia due to stroke; therefore, KEP with MCP may also improve cognitive function in these participants.

On the other hand, MCP did not affect cognitive function compared with baseline scores in our study. One RCT investigated the effect of a multicomponent cognitive intervention in people with mild $\mathrm{AD}[20]$. No significant difference was found for cognitive function compared with a control group in this study. Another RCT performed a 12-month multicomponent cognitive intervention in people with mild to moderate AD; cognitive function had remained stable in the intervention group but had decreased in the control group [19]. However, this trial could not demonstrate that a multicomponent cognitive intervention was effective in improving cognitive function compared with the control group. In our results, we had not assumed that there would be no decline in cognitive measures after 6 months in the MCP group, and would not conclude that the finding of little decline in the MCP only group is attributable to the beneficial effect of MCP.

The physical exercise program which we set in this study is a lower-limb aerobic exercise program using the TERASUERUGO ${ }^{\circledR}$ (Showa Denki Co., Ltd., http://www.showadenki.co.jp/ terasu/product/erugo/erugo75/). The TERASUERUGO ${ }^{\circledR}$ is a newly developed cycle ergometer that can modulate a pedal power of 7 steps, and it is easy to handle. During the study period, no participant experienced muscle or joint pain or fractures due to performing the KEP. Several studies have not shown an effect of lower-limb exercise on cognitive performance in patients with moderate to severe AD [15-17]. A 12-week study, including a lowerlimb exercise program once a week consisting of brisk walking of moderate intensity, strength training, and balance training did not find an effect on cognitive outcome measures [15]. In another study of 6 months, a 30-min walking program performed 4 times a week also did not show a significant change in MMSE scores before and after the intervention period; whereas the control group showed a decrease in MMSE scores during the same periods [17]. Even a 1-year multicenter RCT reported that lower-limb exercise consisting of one session of $1 \mathrm{~h}$ twice a week was not effective in improving behavioral disturbance [16]. In the present study, a significant difference was found in cognitive function between the two groups. It is possible that the physical exercise lasted $1 \mathrm{~h}$ every weekday for 6 months, and the intervention was longer than in other studies [15-17].

According to Sink et al. [8], a physical activity program of moderate intensity, including walking, resistance training, and flexibility exercises (30 min, 3-4 times per week for 24 months), was not effective in improving global or domain-specific cognition compared with a health education program in older, sedentary adults. A recent Cochrane review has also reported no evidence of benefit from exercise on cognition, neuropsychiatric symptoms, or depression [9]. Our results showed that a 6-month KEP with MCP may lead to improved cognitive function compared with MCP. Although this result could not clarify the effect of KEP with MCP because we did not have a control group in our study, a statistically significant difference was found in cognitive function between two groups. Therefore, KEP may have the potential to enhance cognitive function. In this study, the KEP with MCP and the MCP were performed as a part of the standard care treatment because it is better than doing nothing in the nursing home. These interventions are low-cost because they can be performed in a nursing home unlike in a hospital. Moreover, since the participants were residing in the nursing home, the participation rate of residents was $100 \%$. In light of these factors, we believe that our study is practically feasible and replicable within clinical contexts, such as in nursing homes; however, we acknowledge that it may be more difficult to be carried out in a hospital setting.

There were several limitations to this study. First, we did not include a control group receiving placebo treatment, and our results could not clarify the effect of KEP with MCP. 
Kim et al.: Physical Exercise with Multicomponent Cognitive Intervention for Older Adults with Alzheimer's Disease: A 6-Month Randomized Controlled Trial

Second, our results could not demonstrate the effect of KEP alone. Further study is needed to assess the sole effect of KEP. Third, our sample size was small, as it consisted of 33 participants. Therefore, the power of this study was weak. Fourth, the level of functioning was only measured with cognitive scales in this study. Fifth, we did not assess ADL, caregiver burden, and clinicians' observations or impressions. Because of the preliminary nature of this pilot study which included a limited number of participants, we focused on cognitive function only, since general cognitive screening tests are relatively strong correlates of functional status [36]. The validity and sensitivity of the ADAS-cog and MMSE have already been established, and both scales have been used routinely to evaluate general cognitive treatment effects in clinical trials $[37,38]$. Further studies are necessary to investigate the effect of the KEP with MCP on ADL, caregiver burden, and clinicians' observations or impressions of older adults with moderate to severe AD. Lastly, this study was a single trial. Further longitudinal studies of KEP with MCP intervention should include a larger sample and multiple centers.

\section{Conclusion}

A 6-month lower-limb aerobic exercise with MCP consisting of music therapy, art therapy, horticulture therapy, handicraft, recreational therapy, stretching, laughing therapy, and activity therapy might be beneficial for individuals with moderate to severe AD. Our results suggest that lower-limb aerobic exercise may enhance supportive care for cognitive decline in individuals with moderate to severe AD.

\section{Acknowledgments}

We would like to thank all participants and staff from the nursing home 'Dobong Silver Center' for their assistance and participation.

\section{Disclosure Statement}

Dr. M. Kohzuki has received a research grant from Showa Denki Co., Ltd. M.J. Kim, C.W. Han, K.Y. Min, C.Y. Cho, C.W. Lee, Y. Ogawa, and E. Mori indicated no financial or any other kind of personal conflicts with this study.

\section{References}

1 Di Iulio F, Palmer K, Blundo C, Casini AR, Gianni W, Caltagirone C, Spalletta G: Occurrence of neuropsychiatric symptoms and psychiatric disorders in mild Alzheimer's disease and mild cognitive impairment subtypes. Int Psychogeriatr 2010;22:629-640.

-2 Sink KM, Holden KF, Yaffe K: Pharmacological treatment of neuropsychiatric symptoms of dementia: a review of the evidence. JAMA 2005;293:596-608.

-3 Tsoi KK, Hirai HW, Chan JY, Kwok TC: Time to treatment initiation in people with Alzheimer disease: a metaanalysis of randomized controlled trials. J Am Med Dir Assoc 2016;17:24-30.

4 Takeda M, Tanaka T, Okochi M, Kazui H: Non-pharmacological intervention for dementia patients. Psychiatry Clin Neurosci 2012;66:1-7.

5 Olazarán J, Reisberg B, Clare L, Cruz I, Peña-Casanova J, Del Ser T, Woods B, Beck C, Auer S, Lai C, Spector A, Fazio S, Bond J, Kivipelto M, Brodaty H, Rojo JM, Collins H, Teri L, Mittelman M, Orrell M, Feldman HH, Muñiz R: Nonpharmacological therapies in Alzheimer's disease: a systematic review of efficacy. Dement Geriatr Cogn Disord 2010;30:161-178. 
Kim et al.: Physical Exercise with Multicomponent Cognitive Intervention for Older Adults with Alzheimer's Disease: A 6-Month Randomized Controlled Trial

66 Bherer L, Erickson KI, Liu-Ambrose T: A review of the effects of physical activity and exercise on cognitive and brain functions in older adults. J Aging Res 2013;657508:1-8.

7 Paillard T, Rolland Y, de Souto Barreto P: Protective effects of physical exercise in Alzheimer's disease and Parkinson's disease: a narrative review. J Clin Neurol 2015;11:212-219.

8 Sink KM, Espeland MA, Castro CM, Church T, Cohen R, Dodson JA, Guralnik J, Hendrie HC, Jennings J, Katula J, Lopez OL, McDermott MM, Pahor M, Reid KF, Rushing J, Verghese J, Rapp S, Williamson JD; LIFE Study Investigators: Effect of a 24-month physical activity intervention vs. health education on cognitive outcomes in sedentary older adults: the LIFE Randomized Trial. JAMA 2015;314:781-790.

-9 Forbes D, Forbes SC, Blake CM, Thiessen EJ, Forbes S: Exercise programs for people with dementia. Cochrane Database Syst Rev 2015;4:CD006489.

$>10$ Yágüez L, Shaw KN, Morris R, Matthews D: The effects on cognitive functions of a movement-based intervention in patients with Alzheimer's type dementia: a pilot study. Int J Geriatr Psychiatry 2011;26:173-181.

11 Vreugdenhil A, Cannell J, Davies A, Razay G: A community-based exercise programme to improve functional ability in people with Alzheimer's disease: a randomized controlled trial. Scand J Caring Sci 2012;26:12-19.

12 Kemoun G, Thibaud M, Roumagne N, Carette P, Albinet C, Toussaint L, Paccalin M, Dugué B: Effects of a physical training programme on cognitive function and walking efficiency in elderly persons with dementia. Dement Geriatr Cogn Disord 2010;29:109-114.

-13 Arcoverde C, Deslandes A, Moraes H, Almeida C, Araujo NB, Vasques PE, Silveira H, Laks J: Treadmill training as an augmentation treatment for Alzheimer's disease: a pilot randomized controlled study. Arq Neuropsiquiatr 2014;72:190-196.

14 Holthoff VA, Marschner K, Scharf M, Steding J, Meyer S, Koch R, Donix M: Effects of physical activity training in patients with Alzheimer's dementia: results of a pilot RCT study. PLoS One 2015; 10:e0121478.

15 Venturelli M, Scarsini R, Schena F: Six-month walking program changes cognitive and ADL performance in patients with Alzheimer. Am J Alzheimers Dis Other Demen 2011;26:381-388.

16 Rolland Y, Pillard F, Klapouszczak A, Reynish E, Thomas D, Andrieu S, Rivière D, Vellas B: Exercise program for nursing home residents with Alzheimer's disease: a 1-year randomized, controlled trial. J Am Geriatr Soc 2007;55:158-165.

17 Steinberg M, Leoutsakos JM, Podewils LJ, Lyketsos CG: Evaluation of a home-based exercise program in the treatment of Alzheimer's disease: the Maximizing Independence in Dementia (MIND) study. Int J Geriatr Psychiatry 2009;24:680-685.

18 Cotelli M, Manenti R, Zanetti O, Miniussi C: Non-pharmacological intervention for memory decline. Front Hum Neurosci 2012;6:46.

19 Graessel E, Stemmer R, Eichenseer B, Pickel S, Donath C, Kornhuber J, Luttenberger K: Non-pharmacological, multicomponent group therapy in patients with degenerative dementia: a 12-month randomizied, controlled trial. BMC Med 2011;9:129.

20 Buschert VC, Friese U, Teipel SJ, Schneider P, Merensky W, Rujescu D, Möller HJ, Hampel H, Buerger K: Effects of a newly developed cognitive intervention in amnestic mild cognitive impairment and mild Alzheimer's disease: a pilot study. J Alzheimers Dis 2011;25:679-694.

21 Perneczky R, Wagenpfeil S, Komossa K, Grimmer T, Diehl J, Kurz A: Mapping scores onto stages: mini-mental state examination and clinical dementia rating. Am J Geriatr Psychiatry 2006;14:139-144.

22 Schulz KF, Grimes DA: Allocation concealment in randomised trials: defending against deciphering. Lancet 2002;359:614-618.

23 Sakamoto M, Ando H, Tsutou A: Comparing the effects of different individualized music interventions for elderly individuals with severe dementia. Int Psychogeriatr 2013;25:775-784.

24 Hattori H, Hattori C, Hokao C, Mizushima K, Mase T: Controlled study on the cognitive and psychological effect of coloring and drawing in mild Alzheimer's disease patients. Geriatr Gerontol Int 2011;11:431-437.

-25 Jarrott SE, Gigliotti CM: Comparing responses to horticultural-based and traditional activities in dementia care programs. Am J Alzheimers Dis Other Demen 2010;25:657-665.

-26 Tenbrink T, Taylor HA: Conceptual transformation and cognitive processes in origami paper folding. J Probl Solving 2015;8:1-22.

27 Kolanowski A, Fick DM, Buettner L: Recreational activities to reduce behavioural symptoms in dementia. Geriatr Aging 2009;12:37-42.

28 Waryasz GR, McDermott AY: Exercise prescription and the patient with type 2 diabetes: a clinical approach to optimizing patient outcomes. J Am Acad Nurse Pract 2010;22:217-227.

29 Ko HJ, Youn CH: Effects of laughter therapy on depression, cognition and sleep among the community-dwelling elderly. Geriatr Gerontol Int 2011;11:267-274.

30 Politis AM, Vozzella S, Mayer LS, Onyike CU, Baker AS, Lyketsos CG: A randomized, controlled, clinical trial of activity therapy for apathy in patients with dementia residing in long-term care. Int J Geriatr Psychiatry 2004; 19:1087-1094.

-31 Youn JC, Lee DY, Kim KW, Lee JH, Jhoo JH, Lee KU, Ha J, Woo JI: Development of the Korean version of Alzheimer's Disease Assessment Scale (ADAS-K). Int J Geriatr Psychiatry 2002;17:797-803.

32 Kim JM, Shin IS, Yoon JS, Lee HY: Comparison of diagnostic validities between MMSE-K and MMSE for screening of dementia. J Korean Neuropsychiatr Assoc 2002;42:124-130.

-33 Agrell B, Dehun 0: The clock-drawing test. Age Ageing 1998;27:399-403. 
34 Berg K, Wood-Dauphinee S, Williams JI: The balance scale: reliability assessment with elderly residents and patients with an acute stroke. Scand J Rehabil Med 1995;37:27-36.

-35 Ito T, Meguro K, Akanuma K, Ishii H, Mori E: A randomized controlled trial of the group reminiscence approach in patients with vascular dementia. Dement Geriatr Cogn Disord 2007;24:48-54.

-36 Royall DR, Lauterbach EC, Kaufer D, et al: The cognitive correlates of functional status: a review from the Committee on Research of the American Neuropsychiatric Association. J Neuropsychiatry Clin Neurosci 2007; 19:249-265.

37 Mecocci P, Bladström A, Stender K: Effects of memantine on cognition in patients with moderate to severe Alzheimer's disease: post-hoc analyses of ADAS-cog and SIB total and single-item scores from six randomized, double-blind, placebo-controlled studies. Int J Geriatr Psychiatry 2009;24:532-538.

-38 Sabe L, Jason L, Juejati M, et al: Sensitivity and specificity of the Mini-Mental State Exam in the diagnosis of dementia. Behav Neurol 1993;6:207-210. 